\title{
Comment on the hadronic decay of excited heavy quarkonia
}

\author{
Mu-Lin Yan \\ International Center for Theoretical Physics, \\ P.O.Box 586, 34100, Trieste, Italy \\ and \\ Center for Fundamental Physics \\ University of Science and Technology of China \\ Hefei, Anhui 230026, P.R.China* \\ and \\ Yi Wei, Ting-Liang Zhuang \\ Center for Fundamental Physics and Center for Nonlinear Science \\ University of Science and Technology of China \\ Hefei, Anhui 230026, P.R.China \\ January 18, 2020
}

\section{To appear in The European Physical Journal}

\begin{abstract}
We make comments on ref. 迎, and provide partial wave analysis to the decays of excited heavy S-wave $1^{-}$quarkonia into the basic $1^{-}$quarkonia state plus $\pi \pi$. It is revealed that there exist contributions of D-wave transition in $\psi^{\prime} \longrightarrow J / \psi \pi \pi, \Upsilon(2 S) \longrightarrow \Upsilon(1 S) \pi \pi$ and $\Upsilon(3 S) \longrightarrow \Upsilon(1 S) \pi \pi$ by using the data-fitting results in ref.[何. A possible experimental method to measure the $\mathrm{D}$-wave directly is discussed.
\end{abstract}

${ }^{*}$ Mailing Address 
1, Introduction : Starting from the infinite mass limit for the heavy quarkonium, the authors of [1] have presented an interesting systematic derivative expansion for the decays of a heavy excited S-wave spin-1 quarkonium into a lower S-wave spin-1 state in the spirit of chiral perturbation theory. An effective Lagrangian for these exclusive hadronic decays has been constructed in [1]. It is of as follows

$$
\begin{aligned}
\mathcal{L}= & \mathcal{L}_{0}+\mathcal{L}_{\mathrm{SB}} \\
\mathcal{L}_{0}= & g A_{\mu}^{(v)} B^{(v) \mu *} \operatorname{Tr}\left[\left(\partial_{\nu} U\right)\left(\partial^{\nu} U\right)^{\dagger}\right] \\
& +g_{1} A_{\mu}^{(v)} B_{\nu}^{(v) *} \operatorname{Tr}\left[(v \cdot \partial U)(v \cdot \partial U)^{\dagger}\right] \\
& +g_{2} A_{\mu}^{(v)} B^{(v) \mu *} \operatorname{Tr}\left[\left(\partial^{\mu} U\right)\left(\partial^{\nu} U\right)^{\dagger}+\left(\partial^{\mu} U\right)^{\dagger}\left(\partial^{\nu} U\right)\right]+\text { h.c. } \\
\mathcal{L}_{\mathrm{SB}}= & g_{3} A_{\mu}^{(v)} B^{(v) \mu *} \operatorname{Tr}\left[M\left(U+U^{\dagger}-2\right)\right] \\
& +i g^{\prime} \varepsilon^{\mu \nu \alpha \beta}\left[v_{\mu} A_{\nu}^{(v)} \partial_{\alpha} B_{\beta}^{(v) *}-\partial_{\mu} A_{\nu}^{(v)} v_{\alpha} B_{\beta}^{(v) *}\right] \operatorname{Tr}\left[M\left(U-U^{\dagger}\right)\right] \\
& + \text { h.c. }
\end{aligned}
$$

where $U$ is a unitary $3 \times 3$ matrix that contains the Goldstone fields, $M$ is light-quark mass matrix, and $g, g_{1}, g_{2}, g_{3}$ and $g^{\prime}$ are constants. And $A_{\mu}$ is the field of initial S-wave $1^{-}$quarkonium state, $v$ is its velocity vector, $B_{\mu}$ the one of the final $1^{-}$quarkonium state, and $A_{\mu}^{(v)}$ and $B_{\mu}^{(v)}$ are defined by a phase redefinition of $A_{\mu}$ and $B_{\mu}$ respectively. By using this Lagrangian, the authors of ref. [1] have obtained a good fit to the data, especially to $\psi^{\prime} \longrightarrow J / \psi \pi \pi$, $\Upsilon(2 S) \longrightarrow \Upsilon(1 S) \pi \pi$ and $\Upsilon(3 S) \longrightarrow \Upsilon(1 S) \pi \pi$. However, they do not present a correct partial wave analysis to the decay amplitudes. The decays are Swave transition dominant, but in principle $\mathrm{D}$-wave transition is not forbidden, and to search the signals of D-wave in the transitions is meaningful. We will point out in this present comment on [1] that to view the $g_{1}$-term in $\mathcal{L}_{0}$ (eq.(2)) to be a S-wave transition purely is a misunderstanding. We will show that the D-wave transition signals can be seen in the processes of $\psi^{\prime} \longrightarrow J / \psi \pi \pi, \Upsilon(2 S) \longrightarrow \Upsilon(1 S) \pi \pi$ and $\Upsilon(3 S) \longrightarrow \Upsilon(1 S) \pi \pi$. And the ratios between $\mathrm{D}$-wave contributions to the transitions and the total are determined. In addition, we will also propose a possible experiment to measure the D-wave transitions directly, and will discuss how to determine all constans in the process of $A_{\mu} \longrightarrow B_{\mu} \pi \pi$ experimently besides a overall factor.

2, Kinematics and Partial wave analysis: For definiteness, we consider the case of $A_{\mu}=\psi^{\prime}$ and $B_{\mu}=J / \psi$ in this section. In the rest frame of $\psi^{\prime}$, $p_{\pi^{+}}$and $p_{\pi^{-}}$are 4-momentum of $\pi^{+}$and $\pi^{-}$respectively, $q=p_{\pi^{+}}+p_{\pi^{-}}$, $r=p_{\pi^{+}}-p_{\pi^{-}}$and the partial decay rate of $\psi^{\prime}$ into $J / \psi \pi^{+} \pi^{-}$is given by

$$
d \Gamma=A_{\mathrm{ph}}|\mathcal{M}|^{2} d m_{\pi \pi} d \cos \theta
$$


where $\mathcal{M}$ is the decay amplitude,

$$
\begin{aligned}
A_{\mathrm{ph}} & =\frac{1}{2 \pi^{2}} \frac{1}{16 m_{J / \psi}^{2}}\left(q^{2}-4 m_{\pi}^{2}\right)^{\frac{1}{2}}|\vec{q}| \\
|\vec{q}| & =\frac{1}{2 m_{\psi^{\prime}}}\left[\left(m_{\psi^{\prime}}^{2}-\left(m_{\pi \pi}+m_{J / \psi}\right)^{2}\right)\left(m_{\psi^{\prime}}^{2}-\left(m_{\pi \pi}-m_{J / \psi}\right)^{2}\right)\right]^{\frac{1}{2}} \\
q^{2} & =m_{\pi \pi}^{2}=\left(p_{\pi^{+}}+p_{\pi^{-}}\right)^{2}
\end{aligned}
$$

and $\theta$ is the angle between 3-momentum of $\pi^{+}$and 3-momentum of $J / \psi$ in the rest frame of $\pi^{+}-\pi^{-}$. We call $\theta$ as correlation angle hereafter. From the Lagrangian of eq.(1), one can get the transition amplitude $\mathcal{M}$ to the process of $A_{\mu}=\psi^{\prime}$ and $B_{\mu}=J / \psi$ as follows

$$
\begin{aligned}
& \mathcal{M}\left(\psi^{\prime} \rightarrow J / \psi \pi^{+} \pi^{-}\right) \\
= & -\frac{4}{F_{0}^{2}}\left\{\left[\frac{g}{2}\left(m_{\pi \pi}^{2}-2 M_{\pi}^{2}\right)+g_{1}\left(v \cdot p_{\pi^{+}}\right)\left(v \cdot p_{\pi^{-}}\right)+g_{3} M_{\pi}^{2}\right] \varepsilon_{\psi}^{*} \cdot \varepsilon_{\psi^{\prime}}\right. \\
& \left.+g_{2}\left[p_{\pi^{+} \mu} p_{\pi^{-} \nu}+p_{\pi^{+} \nu} p_{\pi^{-} \mu}\right] \varepsilon_{J / \psi^{*}}^{* \mu} \varepsilon_{\psi^{\prime}}^{\nu}\right\}
\end{aligned}
$$

where $\varepsilon_{J / \psi}, \varepsilon_{\psi^{\prime}}$ are the polarization vectors of $J / \psi$ and $\psi^{\prime}$ respectively.

The authors of ref. [1] argued that the $g_{2}$-terms in $\mathcal{M}$ (eq.(5)) are strongly suppressed by the chiral symmetry breaking scale $\Lambda_{\chi S B}$ or heavy quark mass according to their derivative expansion treatment, and they then set $g_{2}=0$. This argument is consistent with the results achieved in [2], [3] and [4] based on the multipole expansion hypothesis for the soft gluon field emission from heavy quarkonium. However this does not mean the D-wave contribution has been excluded. And to view this as pion-D-wave contribution suppression is inadequate, because the $g_{1}$-term has D-wave component also. We show this point in follows.

In the rest frame of $\psi^{\prime}, v=(1,0)$

$$
\left(v \cdot p_{\pi^{+}}\right)\left(v \cdot p_{\pi^{-}}\right)=p_{\pi^{+}}^{0} p_{\pi^{-}}^{0}
$$

Through elementary calculations we get

$$
p_{\pi^{+}}^{0} p_{\pi^{-}}^{0}=A\left(q^{2}\right) P_{0}(\cos \theta)+B\left(q^{2}\right) P_{2}(\cos \theta)
$$

where

$$
\begin{aligned}
& A\left(q^{2}\right)=\frac{1}{4} q^{2}+\frac{1}{6}|\vec{q}|^{2}\left(1+\frac{2 m_{\pi}^{2}}{q^{2}}\right) \\
& B\left(q^{2}\right)=-\frac{1}{2}|\vec{q}|^{2}\left(1-\frac{4 m_{\pi}^{2}}{q^{2}}\right)
\end{aligned}
$$


$P_{0}(\cos \theta)=1, P_{2}(\cos \theta)=\frac{1}{2}\left(\cos ^{2} \theta-\frac{1}{3}\right)$ are Legendre functions. Thus the decay amplitude $\mathcal{M}$ (eq. (5)) can be decomposed into two parts: S-wave $\left(\mathcal{M}_{\mathrm{S}}\right)$ and D-wave $\left(\mathcal{M}_{\mathrm{D}}\right)$. We have

$$
\mathcal{M}=\mathcal{M}_{\mathrm{S}}+\mathcal{M}_{\mathrm{D}}
$$

where

$$
\begin{aligned}
& \mathcal{M}_{\mathrm{S}}=\mathcal{M}_{0}\left\{q^{2}-c_{1}\left(q^{2}+|\vec{q}|^{2}\right)\left(1+\frac{2 m_{\pi}^{2}}{q^{2}}\right)+c_{2} m_{\pi}^{2}\right\} \\
& \mathcal{M}_{\mathrm{D}}=\mathcal{M}_{0}\left\{3 c_{1}|\vec{q}|^{2}\left(1-\frac{4 m_{\pi}^{2}}{q^{2}}\right)\right\} P_{2}(\cos \theta)
\end{aligned}
$$

with

$$
\begin{aligned}
\mathcal{M}_{0} & =\text { const. } \times\left(\varepsilon_{\psi^{\prime}} \cdot \varepsilon_{J / \psi}\right) \\
c_{1} & =-\frac{g_{1}}{3 g}\left(1+\frac{g_{1}}{6 g}\right)^{-1} \\
c_{2} & =2\left(\frac{g_{3}}{g}-\frac{g_{1}}{3 g}-1\right)\left(1+\frac{g_{1}}{6 g}\right)^{-1}
\end{aligned}
$$

The ratio of $\mathrm{D}$-wave transition rate to the total decay rate is defined by

$$
\mathcal{R}_{\mathrm{D}}=\frac{\int d q^{2} \int_{-1}^{+1} d \cos \theta \frac{1}{m_{\pi \pi}} A_{\mathrm{ph}} \sum_{\varepsilon \varepsilon^{\prime}}\left|\mathcal{M}_{\mathrm{D}}\right|^{2}}{\int d q^{2} \int_{-1}^{+1} d \cos \theta \frac{1}{m_{\pi \pi}} A_{\mathrm{ph}} \sum_{\varepsilon \varepsilon^{\prime}}\left|\mathcal{M}_{\mathrm{S}}+\mathcal{M}_{\mathrm{D}}\right|^{2}}
$$

where the limits of $q^{2}$ in the integrals are $q_{\min }^{2}=4 m_{\pi}^{2}, q_{\max }^{2}=\left(M_{\psi^{\prime}}-M_{J / \psi}\right)^{2}$ and the data used in the calculations are $M_{\psi^{\prime}}=3686.0 \mathrm{MeV}, M_{J / \psi}=$ $3096.88 \mathrm{MeV}, M_{\pi}=139.57 \mathrm{MeV}$ and $\sum_{\varepsilon \varepsilon^{\prime}}$ means to sum both up $\varepsilon_{J / \psi}$ and up $\varepsilon_{\psi^{\prime}}$. Thus as long as $g_{1}$ and $g_{3}$ be fixed by fitting the experimental invariant mass spectrum, the contributions of $\mathrm{D}$-wave to the transitions will be determined.

The extensions of the above formulas to the excited $\Upsilon$-decays are straightforward.

3, Ratios of D-wave transition rate to the total decay rate: From eqs.(4) and (5), we obtain the invariant $\pi-\pi$-mass spectrum

$$
\begin{aligned}
\frac{d \Gamma}{d m_{\pi \pi}}= & \int_{-1}^{1} d \cos \theta A_{p h} \sum_{\varepsilon \varepsilon^{\prime}}|\mathcal{M}|^{2} \\
= & \Gamma_{0}|\vec{q}| \sqrt{q^{2}-4 m_{\pi}^{2}}\left\{\left[q^{2}-c_{1}\left(q^{2}+|\vec{q}|^{2}\right)\left(1+\frac{2 m_{\pi}^{2}}{q^{2}}\right)+c_{2} m_{\pi}^{2}\right]^{2}\right. \\
& \left.+\frac{1}{5} c_{1}{ }^{2}|\vec{q}|^{4}\left(1-\frac{4 m_{\pi}^{2}}{q^{2}}\right)^{2}\right\} .
\end{aligned}
$$


where $\Gamma_{0}$ is a constant.

To $\psi^{\prime} \longrightarrow J / \psi \pi \pi$ and $\Upsilon^{\prime} \longrightarrow \Upsilon \pi \pi$, the energies that are available for the pions are small $\left(<586 \mathrm{MeV}<m_{\rho}\right)$. Therefore the fit under chiral limit, i.e., $g_{3}=0$, to these processes is legitimate. This has been done in ref. [1]. The authors of [1] obtained

$$
\begin{aligned}
& \left(\frac{g_{1}}{g}\right)_{c \bar{c}}^{\text {chiral }}=-0.35 \pm 0.03, \text { for } \psi^{\prime} \longrightarrow J / \psi \pi^{+} \pi^{-}, \\
& \left(\frac{g_{1}}{g}\right)_{b \bar{b}}^{\text {chiral }}=-0.19 \pm 0.04, \text { for } \Upsilon(2 S) \longrightarrow \Upsilon(1 S) \pi^{+} \pi^{-} .
\end{aligned}
$$

Substituting eq.(11) and eq.(12) into eq.(9), we obtain the ratios of Dwave transition rate to the total rate for $\psi^{\prime} \longrightarrow J / \psi \pi^{+} \pi^{-}$and $\Upsilon(2 S) \longrightarrow$ $\Upsilon(1 S) \pi^{+} \pi^{-}$respectively as follows

$$
\begin{aligned}
\mathcal{R}_{D}\left(\psi^{\prime} \longrightarrow \psi \pi \pi\right) & =0.065 \pm 0.018 \% \\
\mathcal{R}_{D}(\Upsilon(2 S) \longrightarrow \Upsilon(1 S) \pi \pi) & =0.0156 \pm 0.0078 \%
\end{aligned}
$$

To $\Upsilon(3 S) \longrightarrow \Upsilon(1 S) \pi \pi$, the energies that are available for the pions are not small, and the pions are not soft. Thus the low energy theorem based on the chiral symmetry may be not a good approximation. Consequently, the contributions of $g_{3}$-term turned to be significant. $g, g_{1}$ and $g_{3}$ of this process have been determined [1] to be

$$
\frac{g_{1}}{g}=-2.86 \pm 0.37, \quad \frac{g_{3}}{g}=15.0 \pm 1.2 .
$$

(These values should be checked by a further fit of the correlation angle spectrum. To see the next section.) Then the D-wave content for this process is determined

$$
\mathcal{R}_{D}(\Upsilon(3 S) \longrightarrow \Upsilon(1 S) \pi \pi)=35.5 \pm 14.2 \%
$$

To $\psi^{\prime} \longrightarrow \psi \pi \pi$, in order to reveal the contributions of $g_{3}$-term of eq.(1) ( an effect due to chiral symmetry breaking), the authors have designed a fitting procedure to fit the experimental invariant $\pi-\pi$ mass spectrum. However, it is of a lack of ground in physics. We will show in the next section that the value of $g_{3}$ can be determined by fitting both the invariant mass spectrum and the correlation angle spectrum of this process.

4, Direct measurement to D-wave transition: The fact that there exist a small amount of D-wave transitions in the processes of $\psi^{\prime} \longrightarrow J / \psi \pi \pi$, 
$\Upsilon^{\prime} \longrightarrow \Upsilon \pi \pi$ etc indicates that these decays are not exactly isotropic to $\theta$ distributions. To $\psi^{\prime} \longrightarrow J / \psi \pi \pi$, the $\theta$-distributions can be explored by the correlation angle spectrum of the process, which is as follows

$$
\begin{aligned}
\frac{\mathrm{d} \Gamma}{\mathrm{d} \cos \theta}= & \int_{2 m_{\pi}}^{M_{\psi^{\prime}}-M_{J / \psi}} d m_{\pi \pi} A_{p h}|\mathcal{M}|^{2} \\
= & \text { const. } \Gamma_{0}\left(1+0.18 \mathrm{c}_{2}+0.0085 \mathrm{c}_{2}^{2}-3.54 \mathrm{c}_{1}-0.35 \mathrm{c}_{1} \mathrm{c}_{2}+3.54 \mathrm{c}_{1}{ }^{2}\right. \\
& +\left(0.77 c_{1}+0.077 c_{1} c_{2}-1.62 c_{1}^{2}\right)\left(\cos ^{2} \theta-\frac{1}{3}\right) \\
& \left.+0.20 c_{1}^{2}\left(\cos ^{2} \theta-\frac{1}{3}\right)^{2}\right)
\end{aligned}
$$

A fit to this spectrum represents a direct measurement to the partial waves in the decay.

In the decay amplitude $\mathcal{M}$ without $g_{2}$-terms (eq.(6)), there are two unknown parameters $g_{1} / g$ and $g_{3} / g$. They could be determined by fitting two measured curves: the invariant mass spectrum (eq.(10)) and the correlation angle $(\theta)$ spectrum (eq.(16)). The fit of the former has been performed in [1], and fit of the latter is expected. Because the ratios of D-wave transition rate to the total are generally less than $1 \%$, it is not easy to see the deviations of $\theta$-distribution from the isotropic $\theta$-spectrum. However, it is essential that a great quantity of $\psi^{\prime}\left(\sim 4 \times 10^{6}\right)$ have been accumulated by Beijing Electron Spectrum (BES) on BEPC[5]. This will make the measured curve of $\theta$-spectrum accurate enough to exhibit the the $\mathrm{D}$-wave transition effects in the decay. To $\Upsilon(3 S) \longrightarrow \Upsilon(1 S) \pi \pi$, it is necessary to fit this $\theta$-spectrum in order to check the corresponding result of [1].

Finally, we like to mention that so long as the number of the events are large enough, it is practicable and helpful too to fit the measured bi-variable spectrum of follows

$$
\frac{d^{2} \Gamma}{d m_{\pi \pi} d \cos \theta}=\sum_{\varepsilon \varepsilon^{\prime}} A_{p h}|\mathcal{M}|^{2} .
$$

This is a two-dimensional fit. Because both $m_{\pi \pi}$ and $\theta$ are not integrated out, more interesting informations on the dynamics of the decays are left in this bi-variable spectrum. A full expression of $\mathcal{M}$ is eq.(5) where there are three parameters, $g_{1} / g, g_{2} / g$ and $g_{3} / g$. We like to argue that a full fit to the spectrums of eq.(10), eq.(16) and eq.(17) will provide useful informations for these parameters. Since $g_{2}$-terms in $\mathcal{M}$ (eq.5) describe the physics beyond its leading order effects, the informations on it would be significant to the dynamics.

5, Summary: An interesting systematic derivative expansion for the decays of a heavy excited S-wave spin-1 quarkonium into a lower S-wave spin-1 
state is presented in [1]. In this letter, we make some comments on its results. We provide a partial wave analysis to the decays of excited heavy S-wave $1^{-}$ quarkonia into the basic $1^{-}$quarkonia plus $\pi \pi$. It is revealed that there exist contributions of D-wave transition in $\psi^{\prime} \longrightarrow J / \psi \pi \pi, \Upsilon(2 S) \longrightarrow \Upsilon(1 S) \pi \pi$ and $\Upsilon(3 S) \longrightarrow \Upsilon(1 S) \pi \pi$ by using the data-fitting results in ref. [1]. A possible experimental method to measure the D-wave directly is discussed. It is expected to measure the process of $\psi^{\prime} \longrightarrow \psi \pi \pi$ more precisely by using the data accumulated by BES on BEPC. We argue that through measuring the invariant mass spectrum, the correlation angle spectrum and the bi-variable spectrum precisely, three parameters in the model of [1] could all be determined.

\section{Acknowledgments}

We would like to thank J Li and Z J Guo (BES, Beijing) for helpful discussions. One of us (MLY) wishes to acknoledge the International Center for Theoretical Phsics, Trieste, for its hospitality where part of this work was done. This work was supported in part by the National Funds of China throgh C N Yang, and the Funds of the Institute for High Energy Physics, Beijing.

\section{References}

[1] T. Mannel and R. Urech, Z. Phys. C 73 , 541 (1997)

[2] M. Voloshin and V. Zkharov, Phys. Rev. Lett. 45 ,688 (1981)

[3] V.A. Novikov and M.A.Shifman, Z. Phys. C Particles and Fields, 43 (1981)

[4] T.M.Yan, Phys. Rev. D22, 1652 (1980)

[5] J.Li (BES Collaboration), (private communication). 